\title{
Urgent-Start Peritoneal Dialysis: A Comparison of Catheter Insertion Using Seldinger Technique and Surgical Operation
}

\section{Changqing Yü, Ning Yang\#, Keping Wang, Hongli Lin*, and Longkai Li*}

Department of Nephrology, First Affiliated Hospital of Dalian Medical University, Dalian, China \#These authors contributed fully and equally to this work

*Corresponding authors: Hongli Lin, Department of Nephrology, First Affiliated Hospital of Dalian Medical University, No. 222, Zhongshan Road, Dalian, 116011, China, Tel: +8641183635963-3368; E-mail: linhongli@vip.163.com

Longkai Li, Department of Nephrology, First Affiliated Hospital of Dalian Medical University, No. 222, Zhongshan Road, Dalian, 116011, China, Tel: +8641183635963-3094; E-mail: sdmountaintai@126.com

Received: 01 Nov, 2021 | Accepted: 18 Nov, 2021 | Published: 26 Nov, 2021

Citation: Yu C, Yang N, Wang K, Lin H, Li L (2021) Urgent-Start Peritoneal Dialysis: A Comparison of Catheter Insertion Using Seldinger Technique and Surgical Operation. Int J Nephrol Kidney Fail 7(4): dx.doi.org/10.16966/2380-5498.220

Copyright: (C) 2021 Yu C, et al. This is an open-access article distributed under the terms of the Creative Commons Attribution License, which permits unrestricted use, distribution, and reproduction in any medium, provided the original author and source are credited.

\begin{abstract}
Background: Urgent-Start Peritoneal Dialysis (USPD) is increasingly used worldwide and represents a safe, convenient and cost-effective method to initiate dialysis. However, good catheter insertion techniques are imperative to avoid postoperative complications. While percutaneous catheter insertion by Seldinger technique is considered a safe method with few post-operative complications, comparisons with surgical operation technique are needed, especially in China, where there a few reports on the outcome of percutaneous catheter insertion by Seldinger technique in USPD patients.

Methods: In a retrospective study of 143 USPD patients receiving PD catheter at the Department of Nephrology, First Affiliated Hospital of Dalian Medical University, China, from October 01, 2016 to September 30, 2019, the outcomes of catheter insertions using surgical (S group; $n=83$ ) and percutaneous technique ( $\mathrm{P}$ group; $\mathrm{n}=60$ ) were compared. Duration of operation and break-in periods, post-operative infection and non-infection complications during three-month follow-up, and patient reported outcomes of satisfaction with treatment were documented.

Results: The mean duration of operation and break - in period was significantly longer in S group compared with $\mathrm{P}$ group $(p<0.001)$. Although no significant difference was noted in the post-operative complications between the two groups, there were fewer complications in $P$ group, including peritonitis, exit site infection, and incisional hernia. $55(92 \%)$ patients in P group and $31(37 \%)$ in S group $(p<0.001)$ reported that they were satisfied with short operation duration, less pain and less lying-bed time.

Conclusion: Percutaneous catheter insertion by Seldinger technique was associated with shorter duration of operation and break-in period, fewer post-operative complications, and higher patient-reported satisfaction than surgical insertion. Percutaneous catheter insertion by Seldinger technique is as safe and efficacious as surgical catheter insertion in USPD patients.
\end{abstract}

Keywords: Peritoneal dialysis catheter; Percutaneous insertion; Seldinger technique; Surgical operation

\section{Introduction}

Urgent-start peritoneal dialysis (USPD), defined as initiation of Peritoneal Dialysis (PD) therapy within two weeks after PD catheter insertion [1,2], is increasingly accepted by nephrologists over recent years $[3,4]$ as an effective and cost-saving approach [5] for initiating dialysis treatment in End-Stage Renal Disease (ESRD) patients. As potential concerns of USPD include risk of immediate post-operative complications such as catheter migration and dialysate leakage $[1,6]$ and dialysate leakage occurring within 10 days after catheter insertion [7], good catheter implantation techniques are crucial.

Three catheter insertion techniques are used worldwide, including surgical operation, laparoscopic operation and percutaneous catheter insertion [8]. Since percutaneous catheter insertion is easy to perform, time-saving, less costly and was reported to have less post-operative complications in recent years, it is increasingly used by nephrologists for patients initiating PD $[9,10]$. Percutaneous catheter insertion may be especially useful for those in need of USPD as it is a minimally invasive catheter insertion technique $[11,12]$. However, some adverse effects of percutaneous catheter insertion in USPD patients have been reported, including catheter dysfunction, infection, and fluid leakage, and may have limited its wider use $[8,13,14]$. One reason for conflicting conclusions about the outcome of percutaneous catheter insertion in USPD may be that different operators (nephrologists and surgeons) inserted catheters in the different studies. To avoid the bias of different operators within each study using different techniques, comparisons of outcomes with different catheter insertion techniques should ideally be performed in single center studies where the same operators use both techniques. 
Currently, $\mathrm{PD}$ is receiving increasing attention by China government [15] and in China mainland 86, 344 patients were receiving PD already in 2017 [16]; the population of PD patients can be expected to further increase dramatically during coming years. Surgical operation has since long been the main technique for establishing peritoneal access, but percutaneous catheter insertion is increasingly carried out in China mainland in recent years $[17,18]$. Furthermore, most uremia patients are referred late to peritoneal dialysis and USPD is therefore common in China $[19,20]$. However, while surgical technique was confirmed to be safe in USPD patients, there were few reports on Seldinger technique in China mainland, and, to the best of our knowledge, no reports comparing the outcome of percutaneous catheter insertion and surgical operation in patients needing USPD in China mainland.

Therefore, the aim of the study is to retrospectively analyze collected data about the above-mentioned techniques from one center where USPD was applied in almost all patients (99\%) initiating PD, and to compare the rate of complications between surgical operation and percutaneous catheter insertion performed by the same nephrologists in patients needing USPD.

\section{Methods}

This retrospective study was conducted based on data from patients starting PD from October 01, 2016 to September 30, 2019 at the Department of Nephrology in the First Affiliated Hospital of Dalian Medical University, China. Two senior nephrologists, experienced in catheter insertion both by surgical operation and percutaneous catheter insertion with Seldinger technique, were chosen as operators in the study. All patients who needed USPD (received PD treatment within two weeks after catheter insertion) were identified and followed up in the PD clinic for at least three months to gather data on post-operative outcomes. We excluded those younger than 18 years old, or previously treated by hemodialysis, or not receiving PD treatment within two weeks after catheter insertion, or received catheter insertion in other hospitals. Considering that this was a retrospective study of clinical data, it was not subject to ethical perusal by the local ethics committee.

Collected information in the study included age, sex, cause of ESRD, duration of catheter insertion operation, and break-in periods. The data of the complications after catheter insertion within one month and three months in USPD patients were also collected, including infection complications (peritonitis and exit site infection), non-infection complications (catheter migration, catheter leakage, pleural leakage, scrotal leakage, subcutaneous leakage), and other complications.

All the medical records of these patients were reviewed from the hospital database and the PD center database. All catheter insertions were carried out using either surgical technique or the bedside percutaneous Seldinger technique. The selection of patients for either procedure was generally based on the nephrologists' practices and patient's choice. Straight double-cuffed Tenckhoff catheters were used for all patients. First-generation cephalosporin (cefazolin or cefathiamidine) were administered intravenously half an hour prior to the operation procedure $[8,21]$. All patients were subject to the same procedures before the operation, including washing the whole body, emptying their bladder immediately prior to the procedure and enema one day before the operation [21].

Surgical insertion operation was carried out under local anesthesia by two consultant nephrologists (LL and CY) who together had accumulated experience of more than 600 surgical operations in the past 15 years. Straight double cuffed Tenckhoff catheters were successfully inserted into the pelvic cavity after the peritoneum was incised, and then catheter flow test was performed to confirm the right position of the catheters in the abdominal cavity [7,21]. For percutaneous catheter insertion (also under local anesthesia), penetrating needles were inserted in peritoneal cavity slowly and carefully, 500 to 1000 $\mathrm{ml}$ normal saline was infused in the abdominal cavity through the needles. And then guide wires were inserted through the needles, and the needles were removed out of the abdominal cavity. Next the dilators and split sheath were used to place the catheters into the cavity, and then normal saline was drained out from the catheter to make sure the right position of the catheters according to the drainage volume $[8,22]$.

Data on patient satisfaction were recorded using a simple survey, including pain, duration of operation, and lying-bed time after operation. Satisfaction was defined as less painful complaint, short duration operation ( $<45$ minutes), and less lying-bed time ( $<1$ day). All the data were collected from the hospital database and the PD center database, and the survey result for each patient was recorded as "satisfaction" or "dissatisfaction".

Data were expressed as median with interquartile range (IQR) or percentage, as appropriate. Comparisons between two groups were assessed for nominal variables with Fisher exact tests and assessed for continuous variable with the non-parametric Wilcoxon tests. All statistical analyses were performed using Stata 15.1 (Stata Corporation, College Station, TX, USA). Statistical significance was set at the level of $\mathrm{P}<0.05$.

\section{Results}

There were 146 new PD patients in our center from October 01, 2016 to September 30, 2019. Two patients received catheter insertion in other hospitals, and one patient began PD treatment two weeks later after catheter insertion. Therefore, 143 patients with USPD were included in the study (Figure 1). The percentage of USPD in all the new patients was $99.3 \%$ (143 out of 144 patients) in our center. PD catheters were inserted in 83 patients by using the surgical technique and in 60 patients by using percutaneous Seldinger wire technique. Baseline characteristics of the two groups are shown in table 1 . There were no significant differences in age or gender between the two groups. There were 36 patients with Diabetic Nephropathy (DN) and 26 patients with Chronic Glomerulonephritis (CGN) in Surgical (S) group, and $21 \mathrm{DN}$ patients and $26 \mathrm{CGN}$ patients in Percutaneous (P) group.

Local anesthesia was used in both groups, and there was no difference in anesthetic medication (lidocaine). The mean duration of operation was significantly longer in S group compared with $\mathrm{P}$ group, 58 (53-63) vs 26.5 (22.5-30) minutes; $\mathrm{p}<0.001)$ (Table 1). Also, the break-in period was also longer in $\mathrm{S}$ group compared with $\mathrm{P}$ group [3 (2-3) vs 1 (1-2) days], p <0.001) (Table 1).

No significant difference was found in peritonitis incidence between the two groups. There was one case of peritonitis in the $\mathrm{P}$ group and two cases of peritonitis in the $S$ group within one month $(\mathrm{p}=1.00)$, and there were two cases of peritonitis in each group within three months $(\mathrm{p}=1.00)$. As regards exit site infection, there was only one case in the $S$ group within one month, and no cases in the P group (Table 2).

Non-infection complications are shown in table 2. Mechanical complications occurred in four patients (three in $\mathrm{P}$ group and one in $S$ group; $p=0.31$ ) and were successfully cured, and all patients had normal catheter position after conservative measures. There was no case of catheter migration or subcutaneous leakage in both groups within three months. There were only two cases of catheter leakage in $\mathrm{P}$ group and one case in $\mathrm{S}$ group within one month $(\mathrm{p}=0.57)$, which 


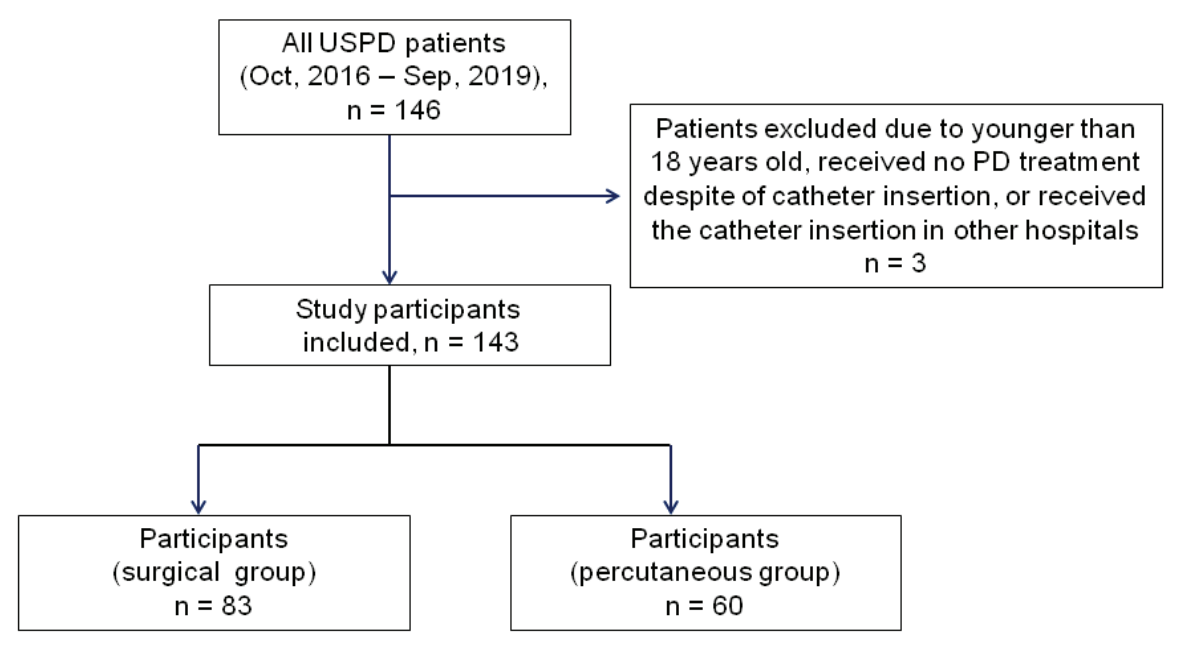

Figure 1: Flow chart of the study.

Table 1: Characteristics of 143 urgent start peritoneal dialysis patients initiating dialysis using percutaneous catheter insertion by Seldinger technique or surgical technique.

\begin{tabular}{|l|c|c|c|}
\hline \multicolumn{1}{|c|}{ Characteristics } & Percutaneous & Surgical & p \\
\hline Number of cases & 60 & 83 & \\
\hline Age (years) & $60(45-69.5)$ & $59(45-70)$ & 0.86 \\
\hline Female $(n, \%)$ & $33(55 \%)$ & $44(53 \%)$ & 0.87 \\
\hline Etiology $(n, \%)$ & & & \\
\hline DN & $20(33.33)$ & $36(43.37)$ & \\
\hline GN & $26(43.33)$ & $26(31.33)$ & \\
\hline BANS & $10(16.67)$ & $16(19.28)$ & \\
\hline Others & $4(6.56)$ & $5(6.02)$ & \\
\hline Duration of operation & $26.5(22.5-30) \mathrm{min}$ & $58(53-63) \mathrm{min}$ & $<0.001$ \\
\hline Break in period & $1(1-2) \mathrm{d}$ & $3(2-3) \mathrm{d}$ & $<0.001$ \\
\hline
\end{tabular}

DN: Diabetic Nephropathy; GN: Glomerulonephritis; BANS: Benign Arterio-Nephrosclerosis.

ceased after reduction of dialysate volume. Only one case of pleural leakage was seen in $S$ group within three months, and there was one case of scrotal leakage in S group within one month and one case of scrotal leakage in $\mathrm{P}$ group within three months. There was no case of wrapped omentum in either group. However, there was one case of incisional hernia in $S$ group within three months but no case of incisional hernia in $\mathrm{P}$ group.

Survey of the satisfaction of patients with operation techniques showed that a larger proportion of patients $(55,92 \%)$ in $P$ group were satisfied with short operation duration, less pain and less lying-bed time compared with the proportion $(31,37 \%)$ in $S$ group $(\mathrm{p}<0.001)$

\section{Discussion}

The outcomes in USPD patients who received of PD catheters using percutaneous catheter insertion by Seldinger technique and
Table 2: Complications at 1 month and 3 months following PD catheter insertion using percutaneous ( $P$ group; $n=60$ ) or surgical ( $S$ group; $n=83$ ) technique in 143 urgent-start PD patients.

\begin{tabular}{|l|c|c|c|c|c|c|c|}
\hline \multirow{2}{*}{ Complications } & \multicolumn{2}{|c|}{$\mathbf{1}$ month } & \multirow{2}{*}{ p } & \multicolumn{2}{c|}{$\mathbf{3}$ month } & \multirow{2}{*}{ p } \\
\cline { 2 - 6 } & $\mathrm{P}$ & $\mathrm{S}$ & & $\mathrm{P}$ & $\mathrm{S}$ & \\
\hline Infection complications \\
\hline Peritonitis & $1(2 \%)$ & $2(2 \%)$ & 1.00 & $2(3 \%)$ & $2(2 \%)$ & 1.00 \\
\hline Exit site infection & 0 & $1(1 \%)$ & 1.00 & 0 & 0 & - \\
\hline \multicolumn{7}{|c|}{ Non-infection complications } \\
\hline Catheter migration & $3(5 \%)$ & $1(1 \%)$ & 0.31 & 0 & 0 & - \\
\hline Catheter leakage & $2(3 \%)$ & $1(1 \%)$ & 0.57 & 0 & 0 & - \\
\hline Pleural leakage & 0 & 0 & 0 & 0 & $1(1 \%)$ & 1.00 \\
\hline Scrotal leakage & 0 & $1(1 \%)$ & 1.00 & $1(2 \%)$ & 0 & - \\
\hline $\begin{array}{l}\text { Subcutaneous } \\
\text { leakage }\end{array}$ & 0 & 0 & - & 0 & 0 & - \\
\hline Other complications & 0 & 0 & - & 0 & $1^{\text {a }}$ & 1.00 \\
\hline
\end{tabular}

P: Percutaneous; S: Surgical; ${ }^{a}$ : Incisional Hernia.

conventional surgical PD insertion technique did not differ in terms of duration of operation, break-in period, and post-operative complications, suggesting that the percutaneous Seldinger technique is a safe and convenient technique. Patients reported greater satisfaction after the percutaneous catheter insertion compared to conventional PD insertion surgery. These results are in line with previous observations of benefits and acceptance of percutaneous catheter insertion [23].

Urgent-start PD appears as a safe and feasible dialysis modality for patients who need dialysis immediately and represents an important opportunity to establish patients with urgent, unplanned dialysis requirements on a cost-effective, home-based dialysis modality with lower serious infection risks than the alternative option of hemodialysis via central venous catheters [1]. USPD is a common mode of dialysis initiation in China, and in our center 99\% of all new PD patients started PD by USPD, reflecting the uremic condition of 
most patients starting on dialysis in our center, and, in general, in China. Complications following percutaneous catheter placement have been considered to be inevitable, particularly leakage during the early days after catheter insertion [7]. However, in our study, only two patients had dialysate leakage in the P group (no statistically significant difference compared with $S$ group) possibly due to the minimal injury in the peritoneum that might not have been fixed as firmly as with the purse-string suture around the opening of peritoneal cavity in surgical operation [8]. Three patients in P group had catheter migration; this is a potential disadvantage of the technique although in our study this problem was resolved after treatment. It is noteworthy that there were few other complications in the P group: few cases of peritonitis, no exit site infection, and no incisional hernia (conceivably due to limited injuries of the abdominal wall with short size of incision). The results from the present study show that percutaneous catheter insertion by Seldinger technique is indeed a reliable and satisfactory technique for patients on USPD.

Although percutaneous insertion by Seldinger technique has been reported to be effective and convenient, it still has some disadvantages compared with surgical operation. For example, it is a blind procedure. During the surgical operation, each organ can be seen clearly around the incision when the abdominal cavity is cut open. Therefore, injuries or perforation of the abdominal organs may rarely occur during conventional surgical operation, while many reports suggested high risk of hurting and even perforating abdominal organs during percutaneous insertion by Seldinger technique despite instillation of radio-contrast dye into the peritoneal cavity in some patients, including bowel perforation [24,25] and bladder injury [26].

Although the current study showed advantages of percutaneous insertion by the Seldinger technique in patients on USPD, there are some limitations that should be considered. First, this is a relatively small retrospective study, and not a randomized clinical trial, and the patients were assigned to receive the different catheter insertion techniques according to the nephrologists or patients' choice. Second, the study may not reflect the real-world situation in many centers because the two nephrologists who performed PD catheter insertions had extensive operation experience. Third, while previous experience in surgical insertion had been obtained for ten years, percutaneous catheter insertion by the Seldinger technique was only carried out for less than two years, and thus the operators were still in the early stage of gathering experience of how to perform percutaneous insertion. With increased experience of percutaneous technique there will be most likely even fewer post-operative complications in USPD patients.

\section{Conclusion}

In conclusion, the present study comparing post-operative complications in USPD patients receiving PD catheter by the Seldinger technique and surgical technique respectively, showed similar outcomes of the two techniques. Our study supports the notion that percutaneous catheter insertion by Seldinger technique is a safe, convenient and efficacious technique for USPD patients. However, further prospective studies in a larger number of patients using a randomized control study design are warranted to confirm the results.

\section{Acknowledgements}

We gratefully acknowledge all the team members from Department of Nephrology, First Affiliated Hospital of Dalian Medical University. This work was supported by [the Natural Science Foundation of Liaoning Province, China \#1] under Grant [number 20180550285]; and [the China Scholarship Council \#2] under Grant [number 201808210378].

\section{Disclosure Statement}

The authors declare that they have no conflicts of interest regarding the publication of this manuscript.

\section{References}

1. Tunbridge $M$, Cho Y, Johnson DW (2019) Urgent-start peritoneal dialysis: is it ready for prime time? Curr Opin Nephrol Hypertens 28: 631-640.

2. Jin H, Ni Z, Mou S, Lu R, Fang W, et al. (2018) Feasibility of UrgentStart Peritoneal Dialysis in Older Patients with End-Stage Renal Disease: A Single-Center Experience. Perit Dial Int 38: 125-130.

3. Wojtaszek E, Grzejszczak A, Grygiel K, Malyszko J, MatuszkiewiczRowinska J (2018) Urgent-Start Peritoneal Dialysis as a Bridge to Definitive Chronic Renal Replacement Therapy: Short- and LongTerm Outcomes. Front Physiol 9: 1830.

4. Alkatheeri AM, Blake PG, Gray D, Jain AK (2016) Success of UrgentStart Peritoneal Dialysis in a Large Canadian Renal Program. Perit Dial Int 36: 171-176.

5. Liu FX, Ghaffari A, Dhatt H, Kumar V, Balsera C, et al. (2014) Economic evaluation of urgent-start peritoneal dialysis versus urgent-start hemodialysis in the united states. Medicine (Baltimore) 93: e293.

6. See EJ, Cho Y, Hawley CM, Jaffrey LR, Johnson DW (2017) Early and Late Patient Outcomes in Urgent-Start Peritoneal Dialysis. Perit Dial Int 37: 414-419.

7. Jo YI, Shin SK, Lee JH, Song JO, Park JH (2007) Immediate initiation of CAPD following percutaneous catheter placement without break-in procedure. Perit Dial Int 27: 179-183.

8. Crabtree JH, Shrestha BM, Chow KM, Figueiredo AE, Povlsen JV, et al. (2019) Creating and Maintaining Optimal Peritoneal Dialysis Access in the Adult Patient: 2019 Update. Perit Dial Int 39: 414-436.

9. Boujelbane L, Fu N, Chapla K, Melnick D, Redfield RR, et al. (2015) Percutaneous versus surgical insertion of pd catheters in dialysis patients: A meta-analysis. J Vasc Access 16: 498-505.

10. Al-Hwiesh AK (2014) Percutaneous peritoneal dialysis catheter insertion by a nephrologist: A new, simple, and safe technique. Perit Dial Int 34: 204-211.

11. Javaid MM, Khan BA, Subramanian S (2019) Is surgical PD catheter insertion safe for urgent-start peritoneal dialysis? Semin Dial 32: 225-228.

12. Abdel-Aal AK, Dybbro P, Hathaway $P$, Guest $S$, Neuwirth $M$, et al. (2014) Best practices consensus protocol for peritoneal dialysis catheter placement by interventional radiologists. Perit Dial Int 34: 481-493.

13. Dombros $N$, Dratwa $M$, Feriani $M$, Gokal R, Heimburger $O$, et al. (2005) European best practice guidelines for peritoneal dialysis. 3 Peritoneal access. Nephrol Dial Transplant 20 Suppl: ix8-ix12.

14. Povlsen JV, Ivarsen P (2006) How to start the late referred ESRD patient urgently on chronic APD. Nephrol Dial Transplant 21 Suppl: ii56-ii59.

15. Yu X, Yang $X$ (2015) Peritoneal dialysis in China: meeting the challenge of chronic kidney failure. Am J Kidney Dis 65:147-151.

16. Tang SCW, Yu X, Chen HC, Kashihara N, Park HC, et al. (2020) Dialysis Care and Dialysis Funding in Asia. Am J Kidney Dis 75: 772-781.

17. Wang H, Wang Y, Zhu J, Chen X, Chen C, et al. (2018) Wang's ForcepsAssisted Percutaneous Insertion and Fixation of Peritoneal Dialysis Catheter. Artif Organs 42: 728-735. 
18. Peng Y, Zhang D, Zheng T, Liu H, Su J, et al. (2019) A half-percutaneous technique for peritoneal dialysis catheter implantation using a modified trocar: A report of 84 cases. Int Urol Nephrol 51: 14511457.

19. Xu D, Liu T, Dong J (2017) Urgent-Start Peritoneal Dialysis Complications: Prevalence and Risk Factors. Am J Kidney Dis 70: 102-110.

20. Ye H, Yang X, Yi C, Guo Q, Li Y, et al. (2019) Urgent-start peritoneal dialysis for patients with end stage renal disease: A 10-year retrospective study. BMC Nephrol 20: 238.

21. Li PK, Szeto CC, Piraino B, de Arteaga J, Fan S, et al. (2016) ISPD Peritonitis Recommendations: 2016 Update on Prevention and Treatment. Perit Dial Int 36: 481-508.

22. Atapour A, Asadabadi HR, Karimi S, Eslami A, Beigi AA (2011) Comparing the outcomes of open surgical procedure and percutaneously peritoneal dialysis catheter (PDC) insertion using laparoscopic needle: A two month follow-up study. J Res Med Sci 16: 463-468.
23. Medani S, Hussein W, Shantier M, Flynn R, Wall C (2015) Comparison of Percutaneous and Open Surgical Techniques for FirstTime Peritoneal Dialysis Catheter Placement in the Unbreached Peritoneum. Perit Dial Int 35: 576-585.

24. Abreo K, Sequeira A (2016) Bowel Perforation during Peritoneal Dialysis Catheter Placement. Am J Kidney Dis 68: 312-315.

25. Shima H, Mizoguchi S, Morine Y, Tashiro M, Okada K, et al. (2018) Intestinal perforation by a peritoneal dialysis catheter in which fungal peritonitis led to diagnosis: A rare case report. CEN Case Rep 7: $208-210$

26. Gülcan E, Sahin SY, Korkmaz M, Kabak O (2018) A Rare Complication during Percutaneous Peritoneal Dialysis Catheter Insertion: Intravesical Placement. Adv Perit Dial 34: 61-63. 